\title{
AGB variables as distance indicators
}

\author{
Michael Feast \\ Astronomy Department, University of Cape Town, Rondebosch 7701, \\ South Africa
}

\begin{abstract}
Evidence for the existence of Mira Period-Luminosity relations is reviewed including recent work on Miras with thick circumstellar shells. The calibration of the relation at $K$ is discussed in detail. The nature of variables deviating from the PL relation and possible metallicity effects are also discussed.
\end{abstract}

\section{Introduction}

The possibility that at least a subset of AGB variables could be used as distance indicators can probably be traced back to the work of Gerasimovic (1928). He suggested on the basis of statistical parallaxes that Mira variables showed a period-(visual) luminosity relation. This was confirmed and extended in later work (e.g. Gyllenberg 1929, 1930; Wilson \& Merrill 1942; Osvalds \& Risley 1961; Clayton \& Feast 1969). However, the scatter in visual absolute magnitude at a given period, though difficult to estimate, seemed quite large (perhaps $\sim 0.5 \mathrm{mag})$. The statistical parallax results were extended to the near infrared and to $M_{b o l}$ by Robertson \& Feast (1981) on the basis of extensive $J H K L$ observations by Catchpole et al. (1979) of nearby Miras.

\section{The slope of the Mira PL relation}

\subsection{The Large Magellanic Cloud}

Glass \& Lloyd Evans (1981) and Glass \& Feast (1982) found that a rather precise period-luminosity relation $\left(\sigma \sim 0.25 \mathrm{mag}\right.$ ) existed at $J H K$ and $m_{\text {bol }}$ on the basis of 11 Miras in the LMC; these relations were further refined by Feast (1984) to include the data of Wood, Bessell \& Paltoglou (1985). This early LMC work depended on only a few infrared observations (often only one) per star.

An extensive programme (Glass et al. 1990) then allowed time-averaged $J H K$ and bolometric magnitudes to be derived for $29 \mathrm{O}$-rich and $20 \mathrm{C}$-rich Miras in the LMC (Feast et al. 1989). At $K$ the PL relation for O-Miras was found to be

$$
M_{K}=-3.47( \pm 0.19) \log P+\beta \text {. }
$$

The standard deviation from this equation is only 0.13 mag. Even though some of this dispersion must be observational, it was found that there was some evidence for a period-luminosity-colour relation. Such a relation is of course to be anticipated since one can hardly expect the Mira instability strip to have no 
width. Recently Glass \& Lloyd Evans (2003) have rederived periods for some of these stars from MACHO data. Their results show that the above relation is rather stable since their rederivation for 26 stars yields a slope of $-3.52 \pm 0.21$, insignificantly different from the above value. In fact the small change is due to the fact that they leave out three stars. It could be argued that at least two of these might well be included as O-Miras (leading to a slope of -3.49 ) since their spectral types (Wood et al. 1985) are K? and S. Note that the designation (M), derived from the photometry, in Feast et al. (1989) implies only a likely $\mathrm{O}$-Mira (i.e. $\mathrm{K}, \mathrm{M}$ or $\mathrm{S}$ ). The $\mathrm{PL}$ relation at $K$ for LMC C-Miras appears to be the same as for the O-Miras.

Feast et al. (1989) also found a PL $\left(M_{b o l}\right)$ relation for LMC O-Miras with a slope of $-3.00 \pm 0.24$. The data of Glass \& Lloyd Evans (2003) gives a slope of $-3.06 \pm 0.26$ (26 stars) or -3.03 (28 stars). In the case of $M_{b o l}$ the slope for the $\mathrm{C}$-Miras is distinctly different $(-1.86 \pm 0.30)$. However, it has never been entirely clear whether this difference is fundamental or due, for instance, to the method of deriving the bolometric magnitudes. This could introduce systematic errors since the spectra of the O- and C-Miras are so different (see also below). A PL slope in $M_{b o l}$ for C-Miras derived by Groenewegen \& Whitelock (1996) using both LMC and Galactic C-Miras of known distance has a slope approximately midway between those for $\mathrm{O}$ - and $\mathrm{C}-\mathrm{Miras}$ just given.

The above discussion refers to Miras with periods shorter than about $420 \mathrm{~d}$. In the sample of Feast et al. (1989) the Miras of longer period fell above a linear extrapolation of the PL relations both at $M_{K}$ and $M_{b o l}$. Hughes \& Wood (1990) chose to fit a PL relation of steeper slope for Miras of periods greater than $400 \mathrm{~d}$. It was later suggested on the basis of approximate theoretical arguments (Willson 2000) that this apparent steepening of the PL relation at longer periods was to be expected. However, the subsets of LMC Miras so far discussed were all established in optical surveys. Thus they are strongly biased to Miras with thin circumstellar shells. In our own Galaxy it is known that Miras with thick circumstellar shells are more frequent amongst the longer-period stars. A series of papers (see references in Whitelock et al. 2003) described the identification of luminous AGB stars in the LMC from IRAS, and their observation at $J H K L$ and with ISO. A subset of these stars are large amplitude (i.e. Mira) variables and these were recently discussed by Whitelock et al. (2003). Extensive $J H K L$ photometry of these stars (including data from Wood et al. 1992 and Wood 1998) allowed the derivation of periods and, together with IRAS and ISO data, bolometric magnitudes. Both O-rich and C-rich objects are present in this sample. For the C-rich objects the periods extend up to $939 \mathrm{~d}$ and for the O-rich variables up to $1393 \mathrm{~d}$. Whitelock et al. (2003) discuss in detail the estimation of the bolometric magnitudes of these stars which can be done in various ways. Whilst there remains uncertainty about the precise bolometric magnitudes of these stars, it is clear that the bulk of those with periods greater than $400 \mathrm{~d}$ fall below the steep PL relation of Hughes \& Wood (1990) and that both the $\mathrm{O}$ - and C-rich objects lie near an extrapolation of the $\mathrm{PL}\left(M_{b o l}\right)$ for O-Miras of shorter period. Whitelock et al. (2003) point out that the long period C-Miras in Magellanic Cloud clusters (Nishida et al. 2000) also fall on an extrapolation of this relation. Extensive mid-infrared work would be required to determine how much of the scatter about the PL relation in the sample of Whitelock et al. (2003) was intrinsic. 
It is known that there are low amplitude semi-regular variables above the Mira PL relation (see e.g. Whitelock 1986, Bedding \& Zijlstra 1998, Wood 2000). Such stars are believed to be evolving towards the Mira PL relation. The nature of the long-period Miras above the PL relation has not been clear. However, Whitelock (see Whitelock \& Feast 2000a and Whitelock et al. 2003) has pointed out that some at least of these Miras are known to have strong lithium lines in their spectra (Smith et al. 1995) and this shows them to be undergoing Hot-Bottom-Burning (HBB). She therefore suggests that this is the distinguishing characteristic of these objects.

It will be clear from the above discussion that considerable caution is required in using Miras with periods greater than $400 \mathrm{~d}$ as distance indicators, especially in optically-selected samples. An example of the problems that can arise is provided by the discovery of a 641-day, large-amplitude variable in the Local Group Irregular galaxy IC1613 (Kurtev et al. 2001). This lies about $0.7 \mathrm{mag}$ above the PL relation at $K$ (for the adopted distance of this galaxy). Suspicion as to its true nature is aroused by the fact that it has a very early spectral type (M3) and is rather blue $(J-K \sim 1.14)$ for its long period. It is possibly a HBB star or, alternatively, a relatively faint supergiant variable.

\subsection{Is the PL slope universal?}

Wood (1995) showed that the PL $(K)$ relation for SMC Miras has a slope close to that in the LMC. Miras in Galactic globular clusters fit the PL $(K)$ slope from the LMC (Feast, Whitelock \& Menzies 2002). In the Sgr I field of the Galactic Bulge, Glass et al. (1995) found that the Miras (all probably O-Miras) fitted a $\mathrm{PL}(K)$ relation of slope $-3.47 \pm 0.35$, i.e. identical to that in the LMC. In this case the scatter about the relation is much higher than in the LMC $(0.35$ mag compared with $0.13 \mathrm{mag}$ ). This difference is primarily due to the depth of the Bulge in the line-of-sight.

In our own Galaxy, Glass et al. (2001) derived periods and mean $K$ magnitudes for 406 Miras in an area $24 \times 24$ arcmin square around the Galactic Centre. There is large and variable extinction in this direction. The general distribution of the stars in the $K-\log P$ plane (their Fig. 4) nevertheless suggests that most of these objects would fit a PL relation with the LMC slope. However, Oritz et al. (2002) used ISO and near IR data to derive bolometric magnitudes for $\mathrm{OH} / \mathrm{IR}$ Miras within half a degree of the Galactic Centre. These stars with relatively thick circumstellar shells show a wide range in luminosity at a given period. Oritz et al. (2002) find that whilst many of their stars lie on a $\mathrm{PL}\left(M_{b o l}\right)$ relation derived from the Sgr I stars of Glass et al. (1995), a considerable number lie below this relation. Thus there appears to be evidence, at least at long period, for Miras below the PL $\left(M_{b o l}\right)$ relation near the Galactic Centre. The matter cannot be considered completely settled yet in view of the considerable difficulty in determining the mean bolometric magnitude of the variables and also in estimating the interstellar extinction. It is worth noting that, if such "subluminous" OH/IR Miras were present in the LMC, they might well have been too faint for existing surveys.

The most striking recent work on the Mira PL slope is that of Rejkuba (2003) who established a well defined PL $(K)$ relation from about 1000 Miras in the giant elliptical galaxy Cen A. The infrared colours of these stars strongly 
suggest that they are mainly O-Miras. Most of these Miras have $\log P$ between 2.3 and 2.7. The slope in Cen A $(-3.37 \pm 0.11)$ clearly agrees well with that in the $\mathrm{LMC},-3.47 \pm 0.19$.

\section{The Mira $\mathrm{PL}(K)$ zero-point}

A realistic determination of the Mira PL zero-point from trigonometrical parallaxes became possible following the Hipparcos astrometric mission. The Hipparcos Mira parallaxes are not of sufficient accuracy to allow their individual use in a determination of both the slope and zero-point of a PL relation. However, since the results summarized above suggest that the $\mathrm{PL}(K)$ slope found for the LMC is similar elsewhere, we can reasonably adopt this slope as applicable to local Galactic Miras. It is then possible to use the method of reduced parallaxes to obtain a zero-point. Whitelock \& Feast (2000b) used this method together with extensive infrared photometry (Whitelock, Marang \& Feast 2000) to derive $\beta$ in equation 1 above for O-rich Galactic Miras.

In Whitelock et al. (2000) (see also Whitelock 2003) it was shown that the shorter-period Miras could be separated by their colours into two sequences, the "short-period-red" and "short-period-blue" stars. These two groups are also distinguished by their kinematics. The latter belong to the main Mira sequence. Whitelock et al. (2000) found evidence from the parallaxes that the "shortperiod-red" Miras were brighter at a given period than those of the main Mira sequence. Whilst the main Mira PL sequence is believed to represent the end points of AGB evolution for stars of different initial masses and/or metallicities, the evolutionary status of the "short-period-red" stars is still uncertain.

Whitelock \& Feast (2000b) found from Hipparcos parallaxes of O-Miras (omitting "short-period-red" stars) that $\beta=0.84 \pm 0.14 \mathrm{mag}$. This result depends on data for 180 Miras though most of the weight resides in a relatively small number of stars. It has recently been suggested that the Hipparcos results for some Miras require a recalibration of the adopted corrections for chromaticity (Platais et al. 2003, Pourbaix et al. 2003). Knapp et al. (2003) have published revised parallaxes based on these new corrections. Using these new parallaxes Whitelock (Leiden Workshop on the Future of AGB Star Research, 2003; and to be published) finds a zero-point of $\beta=1.04 \pm 0.13$ from 140 stars. The change in zero-point is partly due to the fact that the selection of stars is slightly different since Knapp et al. (2003) do not give revised parallaxes for all the Hipparcos Miras. A discussion of statistical bias corrections (Feast 2002) suggests that a small correction of $\sim 0.02 \mathrm{mag}$ is necessary leading to $\beta=1.06$. Table 1 lists this value of the $\mathrm{PL}(K)$ zero-point together with two other Galactic determinations. Vlemmings et al. (2003) have obtained distances to four Mira variables using VLBI astrometry of $\mathrm{OH}$ maser spots and this leads to the value of $\beta$ listed. The final figure in the table is from Miras in globular clusters with cluster distances based on Hipparcos parallaxes of subdwarfs (Feast et al. 2002).

Whitelock \& Feast (2000b) also analysed the parallaxes of Galactic C-Miras. However in view of the uncertainties, which they discuss, it would appear best at present to rely on the LMC result that the (unobscured) C- and O-Miras fit the same $\mathrm{PL}(K)$ relation (Feast et al. 1989). It has already been noted (see 
Table 1. The zero-point of the Mira PL(K) relation

\begin{tabular}{cc}
\hline Method & $\beta$ \\
\hline Parallaxes & $1.06 \pm 0.13$ \\
OH VLBI & $1.01 \pm 0.13$ \\
Globular Clusters & $0.93 \pm 0.14$ \\
& \\
\hline Mean & $1.00 \pm 0.08$ \\
\hline \hline
\end{tabular}

Whitelock et al. 2003) that, within the uncertainties, both the O- and C-rich Miras fit the same $M_{b o l}-\log P$ relation, at least at long periods.

\section{Is the $\mathrm{PL}(K)$ zero-point the same everywhere?}

There are a number of tests that can be applied to see whether the above PL $(K)$ calibration is applicable to all O-Miras.

(1) As Table 1 shows, the values of $\beta$ from Galactic field Miras and those in globular clusters are not significantly different.

(2) The mean value of $\beta$ from Table 1 (1.00) when used with the results of Feast et al. (1989) leads to an LMC modulus of 18.48 with an uncertainty of about $0.1 \mathrm{mag}$ or slightly less. This agrees with an LMC modulus based on Cepheids calibrated in a variety of manners (Feast 2003) which is 18.52 and has a similar small uncertainty.

(3) The difference between the moduli of the SMC and LMC derived from the Mira PL $(K)$ relation is $\sim 0.4 \mathrm{mag}$ (Wood 1995) which compares well with the difference derived from observations of the magnitudes at the red giant tip (0.44 mag, Cioni et al. 2000). A similar result is obtained from Miras in $M_{b o l}$ (Cioni et al. 2003).

(4) The results of Glass et al. (1995) on Miras in the Sgr I field of the Galactic Bulge together with the calibration of Table 1 yield a distance to the Galactic Centre $\left(R_{0}\right)$ of $8.5 \pm 0.7 \mathrm{kpc}$. This may be compared with $8.5 \pm 0.5 \mathrm{kpc}$ derived from Cepheid kinematics (Feast \& Whitelock 1997) and $8.0 \pm 0.4 \mathrm{kpc}$ recently derived from the orbit of a star very close to the central black hole (Eisenhauser et al. 2003).

(5) With the Galactic calibration of Table 1, the Mira $\mathrm{PL}(K)$ results of Rejkuba (2003) for Cen A yield a distance modulus for this giant elliptical of $28.0 \pm 0.2 \mathrm{mag}$, agreeing with the RGB tip distance derived by Rejkuba, $27.9 \pm$ $0.2 \mathrm{mag}$.

These tests indicate that there is no present evidence for any variation of the $\mathrm{PL}(K)$ zero-point, at least within the observational uncertainties.

\section{Are there metallicity effects on the PL relation?}

It is known that, at least in globular clusters, there is a relation between $[\mathrm{Fe} / \mathrm{H}]$ and Mira period (see Feast \& Whitelock 2000). We then need to know whether 
there are variations in chemical abundance at a given period between Miras in different systems. That such seems likely between our Galaxy and the LMC was shown by Glass et al. (1995). They found that the $J H K$ colour-period relations were different in the Sgr I field of the Galactic Bulge from those in the LMC. This question has been discussed by Feast (1996) and Feast \& Whitelock (2000). The Galaxy-LMC difference can be attributed to the weakening of the infrared $\mathrm{H}_{2} \mathrm{O}$ band due to a relative underabundance of oxygen or an overabundance of carbon (locking up oxygen in $\mathrm{CO}$ ) in the LMC. Whether this also implies an overall metal deficiency at a given period in LMC Miras compared with those in our Galaxy is not certain since the $[\mathrm{O} / \mathrm{Fe}]$ ratio for these stars is not known. Whilst the empirical results discussed above indicate that the $\mathrm{PL}(K)$ relation holds in all the places to which it has so far been applied, the colour differences between the Sgr I field and the LMC show that PL relations at some other colours may not be universal. For instance Feast \& Whitelock (1999) point out that that there is a difference in the $\left(K-m_{b o l}\right)$ - $\log P$ relations in the LMC and the Sgr I field. This suggests that a $\mathrm{PL}\left(M_{b o l}\right)$ relation is more metallicity sensitive than a $\mathrm{PL}(K)$ relation, as proposed theoretically by Wood (1990).

\section{Can other AGB variables be used as distance indicators?}

Wood (2000) showed that AGB variables in the MACHO survey of the LMC defined a number of sequences in the $K-\log P$ plane. Similar sequences are found in the Galactic Bulge (Glass \& Schultheis 2003) These sequences show considerable internal scatter. Some of this will be due to the fact that the results depend on only single observations at $K$. However since only the Miras (in Wood's sequence C) are expected to have large $K$ amplitudes (most of the stars are small amplitude semiregular variables) the scatter in the LMC must (except for the Miras) be mainly intrinsic ${ }^{1}$. It remains possible that such sequences might be narrowed by the introduction of a colour term. Even so, it is not at present generally possible to determine to which of Wood's sequences a field SR variable belongs. Thus the use of these sequences for distance determination scems limited for individual stars.

\section{Conclusions}

So far as can presently be determined, the slope and zero-point of the Mira PL relation at $K$ is invariant. The rather precise zero-point of this relation together with the small scatter of individual stars about the relation now makes it possible to derive good distance for Miras in our Galaxy and in the Local Group. The spectacular work of Rejkuba on Miras in Cen A indicates the potential for Mira studies well beyond the Local Group.

Amongst important problems still to solve are: the evolutionary status of the hot bottom burning Miras above the PL relation; the nature of the Miras which apparently lie below the $\mathrm{PL}\left(M_{b o l}\right)$ relation in the region of the Galactic Centre; and the nature of the Galactic "short-period-red" Miras discussed in

\footnotetext{
${ }^{1}$ In the Bulge there is additional scatter due to the depth in the line-of-sight.
} 
Section 3. In addition there is the problem of understanding the small percentage of Miras which show long-term period changes. These have usually been attributed to shell-flashing episodes (Wood \& Zarro 1981) but it has recently been suggested as an alternative that they are due to epochs of envelope relaxation (Zijlstra, Bedding \& Mattei 2002).

Acknowledgments. I am grateful to Patricia Whitelock for her help and advice and to Marina Rejkuba for information in advance of publication.

\section{References}

Bedding, T.R., Zijlstra, A.A. 1998, ApJ, 506, L47

Catchpole, R.M., Robertson, B.S.C., Lloyd Evans, T.H.H., Feast, M.W., Glass, I.S., Carter, B.S. 1979, Sth. Afr. Ast. Obs. Circ. 4, 61

Cioni, M.-R.L., van der Marel, R.P., Loup, C., Habing, H.J. 2000, A\&A, 359, 601

Cioni, M. -R. L. et al. 2003, A\&A, 406, 51

Clayton, M.L., Feast, M.W. 1969, MNRAS, 146, 411

Eisenhauer, F. et al. 2003, astro-ph/0306220

Feast, M.W. 1984, MNRAS, 211, 51p

Feast, M.W. 1996, MNRAS, 278, 11

Feast, M.W. 2002, MNRAS, 337, 1035

Feast, M.W. 2003, in Stellar Candles for the Extragalactic Distance Scale, ed. D. Alloin \& W. Gieren, Lecture Notes in Physics, Springer, in press, astro-ph 0301100

Feast, M.W., Glass, I.S., Whitelock, P.A., Catchpole, R.M. 1989, MNRAS, 241, 375

Feast, M.W., Whitelock, P.A. 1997, MNRAS, 291, 683

Feast, M.W., Whitelock, P.A. 1999 in Post-Hipparcos Cosmic Candles, ed. A. Heck \& F. Caputo, Kluwer, Dordrecht, 75

Feast, M.W. \& Whitelock, P.A. 2000, in The Evolution of the Milky Way, ed. F. Matteucci \& F. Giovannelli, Kluwer, Dordrecht, 229

Feast, M.W., Whitelock, P.A., Menzies J.W. 2002, MNRAS, 329, L7

Gerasimovic, B.P. 1928, Proc. Nat. Acad. Sci. USA, 14, 963 (Harv. Reprint 54)

Glass, I.S., Feast, M.W. 1982, MNRAS, 199, 245

Glass, I.S., Lloyd Evans T. 1981, Nature, 291, 303

Glass, I.S., Lloyd Evans T. 2003, MNRAS, 343, 67

Glass, I.S., Matsumoto, S., Carter, B.S., Sekiguchi, K. 2001, MNRAS, 321, 77

Glass, I.S., Schultheis, M. 2003, astro-ph/0307366

Glass, I.S., Whitelock, P.A., Catchpole, R.M., Feast, M.W., Laney, C.D. 1990, Sth. Afr. Ast. Obs. Circ., No.14, 63

Glass, I.S., Whitelock, P.A., Catchpole, R.M., Feast, M.W. 1995, MNRAS, 273, 383 
Groenewegen, M.A.T., Whitelock, P.A. 1996, MNRAS, 281, 1347

Gyllenberg, W. 1929, Lund Medd. Ser. II, No. 53

Gyllenberg, W. 1930, Lund Medd. Ser. II, No. 54

Hughes, S.M.G., Wood, P.R. 1990, AJ, 99, 784

Knapp, G.R., Pourbaix, D., Platais, I., Jorissen A. 2003, A\&A, 403, 993

Kurtev, R., Geogiev, L., Borissova, J., Li, W.D., Filippenko, A.V., Treffers, R.R. 2001, A\&A, 378, 449

Nishida, S., Tanabé, T, Nakada, Y., Matsumoto, S., Sekiguchi, K., Glass, I.S. 2000, MNRAS, 313, 136

Ortiz, R. et al. 2002, A\&A, 388, 279

Osvalds, V., Risley, A.M. 1961, Publs. Leander McCormick, 11, 147

Platais, I. et al. 2003, A\&A, 397, 997

Pourbaix, D. et al. 2003, A\&A, 399, 1167

Rejkuba, M. 2003, A\&A, in press

Robertson, B.S.C., Feast, M.W., 1981, MNRAS, 196, 111

Smith, V.V., Plez, B., Lambert, D.L., Lubowich, D.A. 1995, ApJ, 441, 735

Vlemmings, W.H.T., van Langevelde, H.J., Diamond, P.J., Habing, H.J.,

Schilizzi, R. 2003, A\&A, 407, 213

Whitelock, P.A. 1986, MNRAS, 219, 525

Whitelock, P.A. 2003 in Mass-Losing Pulsating Stars and their Circumstellar Matter, ed. Y. Nakada, M. Honma \& M. Seki, Kluwer, Dordrecht, 19

Whitelock, P.A., Feast, M.W. 2000a, Mem. S. A. It. 71, 601

Whitelock, P.A., Feast, M.W. 2000b, MNRAS, 319, 759

Whitelock, P.A., Feast, M.W., van Loon, J.Th., Zijlstra, A.A. 2003, MNRAS, 342,86

Whitelock, P.A., Marang, F., Feast, M.W. 2000, MNRAS, 319, 728

Willson, L. E. 2000, ARAA, 38, 573

Wilson, R.E., Merrill, P.W. 1942, ApJ, 95, 248

Wood, P.R. 1990, in From Miras to Planetary Nebulae, ed. M.O. Mennessier \& A. Omont, Editions Frontières, Gif-sur-Yvette, 67

Wood, P.R., 1995, in ASP Conf. Ser., Vol. 83, Astrophysical Applications of Stellar Pulsation, ed. R.S. Stobie \& P.A. Whitelock, (San Francisco: ASP), 127

Wood, P.R. 1998, A\&A, 338, 592

Wood, P.R. 2000, Publ. Ast. Soc. Aust. 17, 18

Wood, P.R., Bessell, M.S., Paltoglou, G. 1985, ApJ, 290, 477

Wood, P.R., Whiteoak, J.B., Hughes, S.M.G., Bessell, M.S., Gardner, F.F., Hyland A.R. 1992, ApJ, 397, 552

Wood, P.R., Zarro, D.M. 1981, ApJ, 247, 247

Zijlstra, A.A., Bedding, T.R., Mattei, J.A. 2002, MNRAS, 334, 498 


\section{Discussion}

Dambis: Should the optical PL relation be corrected for a colour term, or perhaps be more metallicity dependent?

Feast: Optical PL relations have considerable intrinsic width and are currently of little usefulness. One might expect them to be metallicity dependent, but it is not known if this is so.

Chambliss: Please comment on bolometric corrections for these very cool stars. How certain are these and the derived $M_{b o l}$ data that you use?

Feast: The results do not depend on the use of bolometric corrections, but on direct integration of the energy distribution. The uncertainties in the fluxes at different wavelengths and their effect on $M_{b o l}$ are discussed in detail in Whitelock et al. (2003, MNRAS, 342, 86).

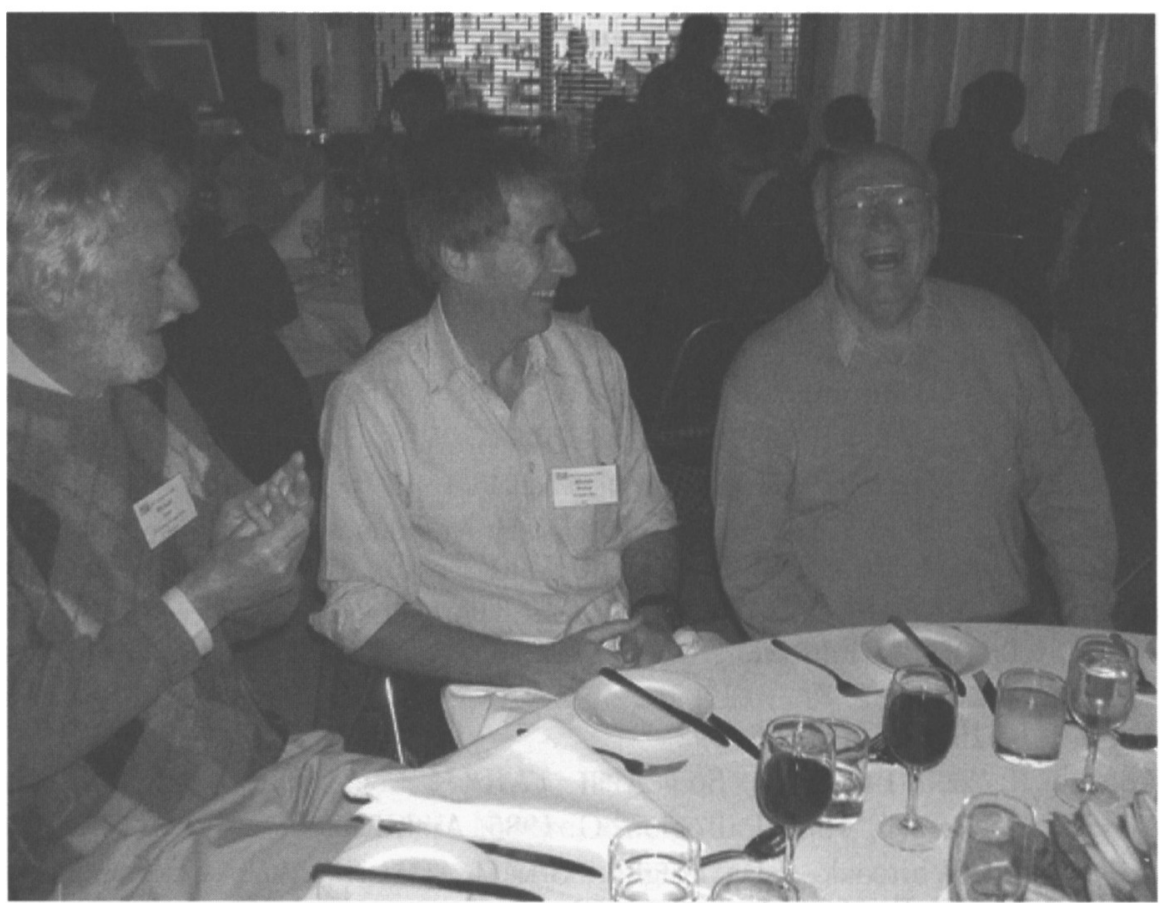

Micheal Feast, Alistair Walker and Harold McNamara 